\title{
Glia: silent partners in energy homeostasis and obesity pathogenesis
}

\author{
John D. Douglass ${ }^{1}$ • Mauricio D. Dorfman ${ }^{1}$ • Joshua P. Thaler ${ }^{1}$
}

Received: 1 August 2016 / Accepted: 11 November 2016/Published online: 16 December 2016

(C) Springer-Verlag Berlin Heidelberg 2016

\begin{abstract}
Body weight stability requires homeostatic regulation to balance energy intake and energy expenditure. Research on this system and how it is affected by obesity has largely focused on the role of hypothalamic neurons as integrators of information about long-term fuel storage, shortterm nutrient availability and metabolic demand. Recent studies have uncovered glial cells as additional contributors to energy balance regulation and obesity pathogenesis. Beginning with early work on leptin signalling in astrocytes, this area of research rapidly emerged after the discovery of hypothalamic inflammation and gliosis in obese rodents and humans. Current studies have revealed the involvement of a wide variety of glial cell types in the modulation of neuronal activity, regulation of hormone and nutrient availability, and participation in the physiological regulation of feeding behaviour. In addition, one glial type, microglia, has recently been implicated in susceptibility to diet-induced obesity. Together, these exciting new findings deepen our understanding of energy homeostasis regulation and raise the possibility of identifying novel mechanisms that contribute to the pathogenesis of obesity.
\end{abstract}

Keywords Astrocytes · Brain · Central nervous system · Diabetes · Energy homeostasis · Glia $\cdot$ Hypothalamus · Inflammation $\cdot$ Microglia $\cdot$ Obesity $\cdot$ Review

Electronic supplementary material The online version of this article (doi:10.1007/s00125-016-4181-3) contains a slideset of the figures for download, which is available to authorised users.

Joshua P. Thaler

jpthaler@uw.edu

1 UW Diabetes Institute and Department of Medicine, University of Washington, 850 Republican St, S248, Box 358055,

Seattle, WA 98109, USA

\author{
Abbreviations \\ AHA American Heart Association \\ ALS Amyotrophic lateral sclerosis \\ BBB Blood-brain barrier \\ CNS Central nervous system \\ CX3CL1 C-X3-C motif chemokine ligand 1 \\ CX3CR1 C-X3-C motif chemokine receptor 1 \\ DIO Diet-induced obesity \\ DREADDs Designer Receptors Exclusively Activated by \\ Designer Drugs \\ GLP-1 Glucagon-like peptide 1 \\ HFD High-fat diet
}

\section{Introduction}

Obesity is a significant public health problem since it greatly increases the risk of highly morbid chronic diseases, including type 2 diabetes, hypertension, cardiovascular disease and many cancers. Since 1980, the worldwide prevalence of obesity has nearly doubled with Western countries leading the way. Currently, over half of adults in the European Union and two-thirds in the USA are overweight or obese (BMI $>25 \mathrm{~kg} / \mathrm{m}^{2}$ ). Nevertheless, in both lean and obese individuals, fat mass generally remains stable over long periods of time due to the homeostatic matching of energy intake and expenditure [1]. Furthermore, body weight and adiposity are $>50 \%$ attributable to inherited predisposition [2]. Given that obesity has a strong genetic component and does not involve failure of energy homeostasis, how does an obesity epidemic arise? Currently, the leading hypothesis posits that environmental pressures of the modern world, such as the consumption of highly palatable diets, elevate the defended level of fat mass in susceptible individuals. Unfortunately, efforts to reverse this process pharmacologically have been largely met with failure 
because of our continued limited understanding of how diet and other environmental factors affect the neurocircuitry and molecular mediators of energy homeostasis. Despite great advances in this field since the discovery of the adiposity hormone, leptin, over 20 years ago [3], obesity pathogenesis remains an enigma perhaps in part because of the exclusive focus on the role of neurons in disease progression. In fact, in other brain diseases such as amyotrophic lateral sclerosis (ALS), Alzheimer's disease and multiple sclerosis (MS), glial cells are significant contributors to pathologic processes, with neurons being the targets of toxic or protective cell-cell interactions [4]. Since our discovery that hypothalamic gliosis was associated with inflammation resulting from high-fat diet (HFD) feeding [5], the field has expanded quickly; many discoveries of new functions for non-neuronal cells in metabolic regulation have since been reported [6]. In this review, we provide a brief synopsis of hypothalamic inflammation (reviewed extensively elsewhere [7, 8]), describe the basic glial cell types, highlight glial cell contributions to the physiological and pathological regulation of energy homeostasis, and discuss the translational implications of these findings.

\section{Hypothalamic inflammation}

The hypothalamus is a ventral forebrain structure comprised of over ten discrete nuclei surrounding the third cerebral ventricle. It regulates many homeostatic functions including temperature, circadian rhythms, reproductive behaviour and energy balance. Its location and structure provide the unique capacity for both neural connectivity with other brain regions and hormonal output through a specialised vasculature shared with the pituitary gland. In addition, this anatomical arrangement allows subsets of neurons (largely within the arcuate nucleus of the mediobasal hypothalamus) to sense circulating indicators of energy availability including nutrients (e.g. glucose, fatty acids, amino acids), adiposity hormones (e.g. leptin, insulin) and appetite modulators (e.g. ghrelin, glucagonlike peptide 1 [GLP-1]) [9]. Integrating these signals, the arcuate neurons adjust metabolic rate and feeding behaviour to maintain energy homeostasis. Since body weight stability depends on the fidelity of this system, there has been great interest in identifying factors that impair this function to reveal mechanisms underlying obesity pathogenesis.

HFD promotes hypothalamic inflammation The discovery of inflammatory mediators in the hypothalamus of rodents in response to HFD feeding provided just such candidate factors that could disrupt energy homeostasis [10]. Prior studies of peripheral metabolic tissues, such as liver and adipose tissue, have established that chronic low-grade inflammation resulting from dietary overconsumption interferes with insulin signalling [11-13], leading to a state of glucose intolerance. Similarly, hypothalamic inflammation in HFD-fed rodents (as well as non-human primates and other rodent models, including insulin-deficient and fructose-fed rats [5, 6, 14-19]) provides an analogous mechanism to explain the impairment of energy balance with diet-induced obesity (DIO). Akin to peripheral tissue inflammation, HFD-induced hypothalamic inflammation triggers the activation of stress kinases, such as c-Jun Nterminal kinase (JNK) and the IKB kinase (IKK), that inhibit downstream mediators of the insulin and leptin pathways $[10,19]$. This leptin/insulin resistance in critical hypothalamic neurons increases the homeostatic body weight set point, ultimately resulting in obesity [19].

Hypothalamic inflammation and DIO Since the initial description by De Souza et al [10], considerable evidence has accumulated for a functional contribution of hypothalamic inflammation to DIO susceptibility. First, hypothalamic inflammation develops within the first days of HFD exposure, prior to significant weight gain and concomitant with the onset of leptin resistance and upregulation of neuronal stress markers [5, 20]. Furthermore, mouse models with genetically diminished inflammatory signalling in hypothalamic neurons show reduced HFD-associated hyperphagia and weight gain as a consequence of preserved leptin and insulin sensitivity $[19,21]$. Likewise, female C57BL6 mice are relatively resistant to both hypothalamic inflammation and HFD-induced obesity (Fig. 1) [18, 22]. Nevertheless, not all data are supportive of this causal relationship; a number of models (e.g. leptin-deficient $o b / o b$ mice, chronic ghrelin-treated rats, foodrestricted DIO rats) show dissociations between obesity susceptibility and hypothalamic inflammation status [23-25], suggesting that the connection between obesity and hypothalamic inflammation may depend on the interplay between a hyperenergetic environment, weight gain and neuroendocrine pathways, among other factors. Moreover, HFD feeding is associated with an accumulation of activated glial cells including astrocytes and microglia, similar to the obesity-associated tissue infiltration in the periphery by activated immune cells (primarily macrophages but also virtually all other leucocyte classes) [11-13]. Thus, as discussed below, determining the contributions of different glial cell types to energy balance will help clarify the role of hypothalamic inflammation in metabolic disease and potentially identify novel mechanisms underlying obesity pathogenesis.

\section{Glial cell types}

The discovery of glia In 1906, the brilliant Spanish histopathologist Santiago Ramon y Cajal won the Nobel prize together with the Italian scientist Camillo Golgi for their research on mapping the nervous system, from which Cajal coined 'The 
Fig. 1 The degree of microgliosis in response to HFD feeding predicts metabolic outcome. (a) HFD-fed male mice and rats have an accumulation and activation of microglia and astrocytes (not shown) in the hypothalamus that correlates with weight gain. (b) Hypothalamic depletion of microglia in male mice using PLX5622, a CSF1R inhibitor, decreases food intake in the context of high saturated fatty acid consumption. (c) Females are relatively resistant to diet-induced hypothalamic gliosis and obesity. (d) Female mice with $\mathrm{Cx} 3 \mathrm{crl}$ deficiency (encoding the chemokine (C-X3-C motif) receptor 1 [CX3CR1]) manifest male-pattern hypothalamic microglial accumulation and activation, accompanied by a marked increase in DIO susceptibility (M. Dorfman, J. Douglass, J. Thaler, unpublished observation). $3 \mathrm{~V}$, third ventricle

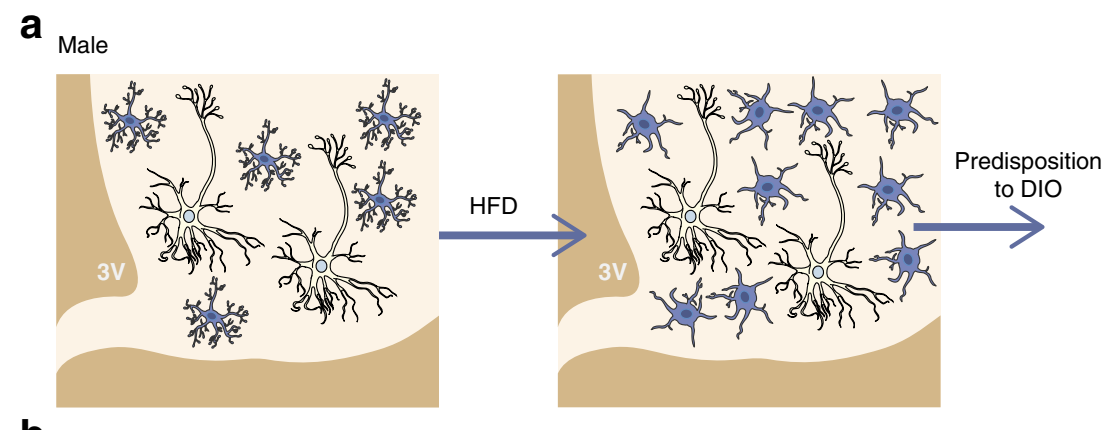

$\mathbf{b}_{\text {Male }}$

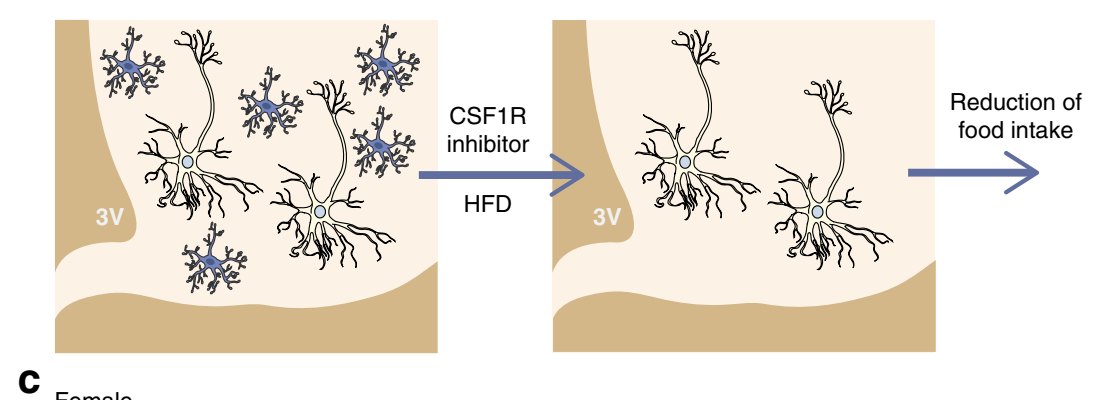

C Female

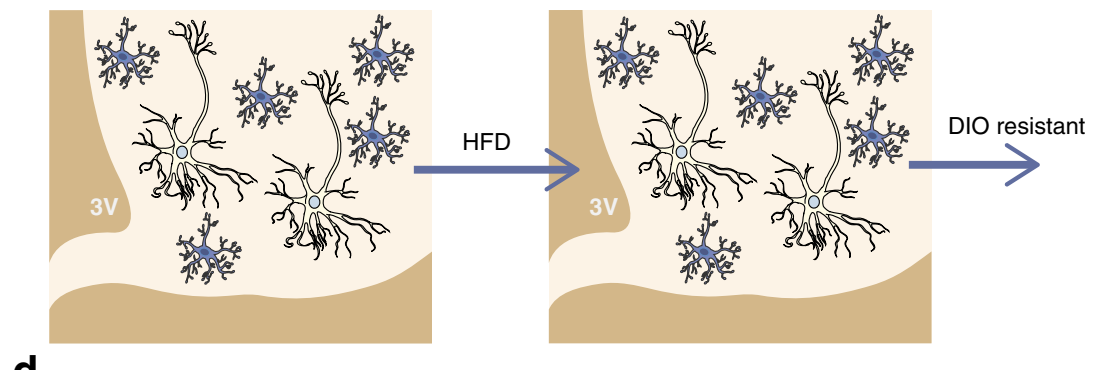

$\mathbf{d}_{\text {Female }}$

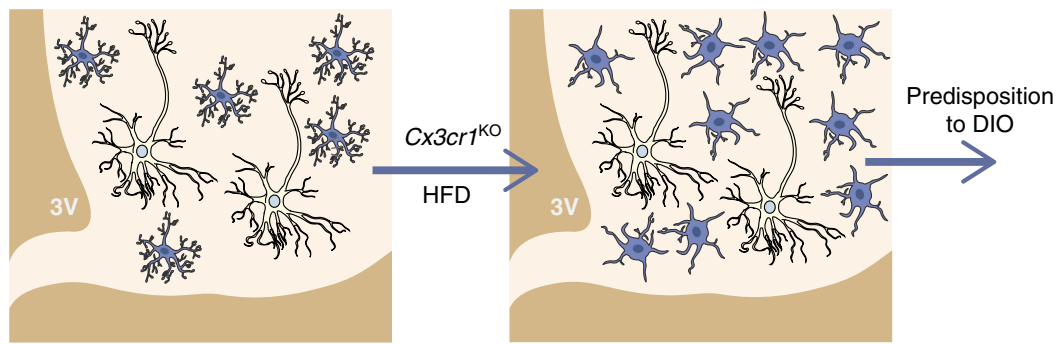

Neuron Doctrine'. This concept states that the nervous system is comprised of individual cells that are interconnected to provide the basis for its function. Cajal had also carefully documented the presence of an even more numerous population of unconnected cells (named 'glia' for glue by Rudolf Virchow in 1856). Like Virchow before him (and over 50 years of scientists after him), Cajal largely ignored these cells, viewing them only as providers of structural support for the central nervous system (CNS) or insulation for neuronal projections. With the early confirmation of these rudimentary properties, the study of glial cells lagged severely behind other areas of neuroscience for nearly a century after their discovery. Only in the past 20 years have we begun to recognise the full breadth and complexity of glial cells, which include astrocytes, microglia, tanycytes, polydendrocytes, and oligodendrocytes (Table 1). From synaptic formation and pruning, to neurovascular coupling and phagocytosis, glia play critical roles in CNS activity during development, health and disease [26]. Until recently, however, most studies that focused on glia were restricted to use of cultured cells or descriptive analyses because of a lack of cell-specific tools.

The functional era for glial research From the year 2000, research on glial biology moved into the functional era, with the development of the astrocyte-specific human glial fibrillary acidic protein ( $h G F A P)-C r e$, and oligodendrocyte- 
Table 1 Cell types of the CNS

\begin{tabular}{|c|c|c|}
\hline Cell type & Cell markers & Cell functions \\
\hline \multicolumn{3}{|l|}{ Neurons } \\
\hline & $\begin{array}{l}\text { Nestin } \\
\text { Synapsin }\end{array}$ & $\begin{array}{l}\text { Information processing } \\
\text { Sensory input integration } \\
\text { Behavior modulation }\end{array}$ \\
\hline \multicolumn{3}{|l|}{ Astrocytes } \\
\hline & $\begin{array}{l}\text { GFAP } \\
\text { ALDH1L1 } \\
\text { GLAST }\end{array}$ & $\begin{array}{l}\text { Nutrient support } \\
\text { Neurotransmitter reuptake } \\
\text { Neurovascular coupling } \\
\text { Gliotransmitter production }\end{array}$ \\
\hline
\end{tabular}

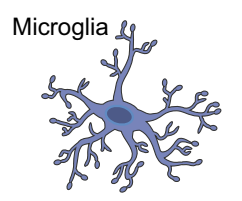

\section{$\mathrm{CD} 11 \mathrm{~b}$} CX3CR1 IBA1

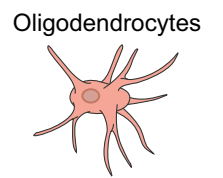

$$
\begin{aligned}
& \text { O4 } \\
& \text { MOG } \\
& \text { MBP }
\end{aligned}
$$

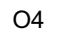

\section{Polydendrocyte} (NG2 cells)

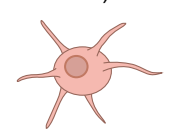

PDGFRa

NG2

SOX10

Tanycytes

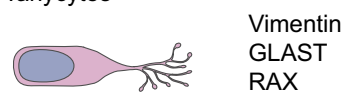

\section{Neurogenesis \\ BBB integrity}

Nutrient import to CNS

Synaptic pruning

Cellular debris phagocytosis

Cerveillance

Inflammation

Myelination

Trophic support for neurons

Oligodendrocyte precursor

Neurogenesis

BBB integrity

ALDH1L1, aldehyde dehydrogenase 1 family member L1; GFAP, glial fibrillary acidic protein; GLAST, glutamate aspartate transporter; GLT-1, glutamate transporter 1; IBA1, ionised calcium-binding adapter molecule 1 ; MBP, myelin basic protein; MOG, myelin oligodendrocyte glycoprotein; NG2, nerve/glia antigen 2; O4, oligodendrocyte marker; PDGFR $\alpha$, platelet-derived growth factor receptor alpha; RAX, retina and anterior neural fold homeobox transcription factor; SOX10, SRY-related HMGbox 10

specific myelin basic protein $(M b p)$-Cre transgenic mice. Recently, this has been followed by the development of polydendrocyte ( $N G 2$, also known as $C S P G 4)$, tanycyte $($ Rax $)$ and microglial $(\mathrm{Cx} 3 \mathrm{cr} 1)$-expressing mouse lines (Table 1; reviewed in [27-29]). In addition, a number of sophisticated novel methods used to manipulate neuronal activity using light (optogenetics) [30] or designer drugs (chemogenetics) [31] have been adapted for glial experimentation. Importantly, the first experiments using these methods to study the role of glial cells in metabolism were reported in 2015 (see below) [32]. With the availability of these new techniques and animal models, the past few years have seen remarkably rapid progress towards identifying and characterising the function of glial cells in energy homeostasis regulation and their potential contribution to the pathogenesis of obesity.
Microglial activation and obesity pathophysiology

Microglia: myeloid cells of the CNS The similarities between peripheral and hypothalamic inflammation during DIO suggested the possible involvement of CNS immune cells in mediating the inflammatory response, analogous to the role of macrophages in the periphery. Since the brain is an immune-privileged structure with a cell-impermeable barrier, immune surveillance and function must be maintained exclusively by CNS-specific cells called microglia (named by Cajal, based on their small size) [33]. Microglia are unique among cells of the CNS because of their myeloid characteristics and distinct ontogeny. However, unlike peripheral myeloid cells, they originate and migrate from the yolk sac rather than bone marrow [34] and are locally renewed throughout adult life [35]. In addition, these cells have a unique transcriptomic signature, expressing a number of immunerelated genes such as the purinoreceptor P2y12, Tmem 119 and $\mathrm{Cx} 3 \mathrm{crl}$. These genes distinguish microglia from all other CNS cells and most monocytes/macrophages outside of the CNS [36].

Microglia act as first responder cells to a variety of CNS insults, including pathogens, ischaemia and neuron injury. They are rapidly recruited to regions of damage or cell death where they proliferate in situ and convert from a surveillant, highly ramified morphology to an amoeboid activated form [33]. Activated microglia perform phagocytic functions and produce proinflammatory mediators including cytokines, chemokines, reactive oxygen species and nitric oxide, together serving to neutralise threats and maintain a neuroprotective milieu. However, prolonged microglial activation may result in chronic inflammation that contributes to the progression of neurodegenerative and neoplastic diseases [37].

Microglia and energy homeostasis As described previously, HFD feeding is associated with the onset of hypothalamic inflammation within 3 days of diet exposure [5]. Over the same time frame, activated microglia accumulate in the mediobasal hypothalamus, possibly mediating the inflammatory response (Fig. 1a) [5]. Consistent with this hypothesis, the expression of proinflammatory genes is increased in hypothalamic microglia extracted from mice exposed to HFD for periods of 3 days up to, at least, 3 months [6, 38]. Furthermore, ablation of microglia using in vivo administration of colony stimulating factor 1 receptor (CSF1R) antagonists eliminates hypothalamic inflammation and signs of neuronal stress (Fig. 1b) [6]. Despite these findings, however, little is known about the role of microglia in metabolic regulation.

Based on two reports using the microglial ablation mouse model, and a separate study using rats that had received a central infusion of the microglial-selective inhibitor minocycline, microglia do not make measurable contributions to body weight 
or food intake regulation under chow-fed conditions $[6,39,40]$. However, if the same microglia-depleted mice are gavage-fed with a saturated fat solution in addition to their regular chow, they reduce their ad libitum chow intake (Fig. 1b) [6], suggesting microglial activation may interfere with nutrient sensing by the hypothalamus. Overall, these initial studies imply the presence of signals (proinflammatory or otherwise) produced by activated microglia that modulate or impair neuronal regulation of energy homeostasis. Consistent with this view, Reis et al reported that microglia mediate the effect of the proinflammatory molecule, endotoxin (also known as lipopolysaccharide [LPS]), to alter neuronal activity of hypothalamic neurons [40]. Efforts by our research group are currently underway to identify these molecular mediators of microglia-neuron crosstalk.

Regulation of microglial activity As described above, microglia have a destructive potential that can promote neurotoxicity. To limit this possibility, neurons produce inhibitory factors such as the chemokine $\mathrm{C}-\mathrm{X} 3-\mathrm{C}$ motif chemokine ligand 1 (CX3CL1, also known as fractalkine), which silences neighbouring microglia through its cognate inhibitory Gprotein-coupled receptor $\left(\mathrm{G}_{\mathrm{i}}\right.$-coupled receptor), $\mathrm{C}-\mathrm{X} 3-\mathrm{C}$ motif chemokine receptor 1 (CX3CR1) (Table 1) [41, 42]. Accordingly, Cx3crl deletion in mice causes excess microglial activation and increased susceptibility to CNS inflammatory diseases [43]. Surprisingly, however, mice with deficient CX3CL1-CX3CR1 signalling show largely unchanged metabolic variables or mild reductions in DIO susceptibility [44-48], perhaps due to peripheral, as well as central effects [36, 49-52]. Alternatively, these results may reflect the exclusive focus on male mice, which develop HFDinduced microglial activation irrespective of CX3CR1 status $[6,18]$. In contrast, female mice are relatively resistant to hypothalamic inflammation, microgliosis and DIO (Fig. 1c) $[18,22]$ and, therefore, may be sensitive to loss of CX3CR1. Indeed, we have found that female CX3CR1 knockout models lose their protection from DIO with 'male-type' inflammation and microglial activation in the hypothalamus (M. Dorfman, J. Douglass, J. Thaler, unpublished results; Fig. 1d). Future studies using microglial-specific targeting methods should help delineate the role of inflammatory signalling in the pathogenesis of obesity and clarify the metabolic function of microglial receptors with regards to body weight-regulating hormones, such as leptin, GLP-1 and oestrogen.

\section{Astrocyte-neuron interactions in energy homeostasis}

\section{Astrocytes provide metabolic support to neurons}

Astrocytes are large, star-shaped glia that are broadly distributed throughout the CNS and considered its most abundant cell type. They perform many essential homeostatic roles in the brain, including regulation of synaptic transmission, maintenance of the blood-brain barrier (BBB), and fluid and ion homeostasis [53]. The anatomical distribution of astrocytes places their processes in close proximity to the blood vessels that supply neurons, facilitating their ability to control the import of circulating hormones and metabolites, and to regulate neurovascular coupling. In the hypothalamus, this spatial relationship is particularly important for neuronal control of systemic glucose homeostasis. Notably, insulin receptor deletion in hypothalamic astrocytes reduces glucose and insulin uptake across the BBB, in turn impairing peripheral glucose tolerance [54]. Thus, alterations to CNS metabolism, generated by the gatekeeper function of astrocytes, induce reciprocal changes in body fuel homeostasis.

Astrocytes participate in synaptic activity, supplying energy substrates to neurons, secreting gliotransmitters (such as adenosine) and terminating signalling through glutamate reuptake (Fig. 2) [55]. As part of their role of providing metabolic support to neurons, astrocytes possess the bulk of CNS glycogen stores and can mobilise this energy supply in response to decreases in local glucose levels [56, 57]. This local source of fuel in the form of lactate (Fig. 2) has been shown to be critical for neuronal function during hypoglycaemia [58] and periods of high synaptic activity [59-62]. Moreover, astrocytes are also a primary source of CNS-derived ketone bodies (Fig. 2) and their production in the hypothalamus of HFD-fed rodents stimulates adjacent anorexigenic neurons to reduce energy intake [63, 64].

\section{Astrocyte contributions to energy homeostasis in health} There is accumulating evidence that astrocytes modulate energy homeostasis under physiological conditions. Activation of hypothalamic astrocytes by leptin modifies connections with adjacent neurons [65, 66] (Fig. 2). Accordingly, deletion of the leptin receptor in astrocytes results in remodelling of the hypothalamic neuronal circuitry and diminished leptininduced anorexia [67-70], highlighting how interactions between the glial and neuronal network may affect the response to satiety cues. Other hormonal signals such as ghrelin and GLP-1 can also activate astrocytes, but more work is needed to firmly establish the in vivo significance of these findings [71, 72]. Finally, using Designer Receptors Exclusively Activated by Designer Drugs (DREADDs), a chemogenetic approach to modulate the activity of specific cells, Yang et al demonstrated that hypothalamic astrocytes suppress food intake via adenosine-mediated inhibition of the orexigenic Agouti-regulated protein (AgRP) neurons (Fig. 2) [32]. However, a more recent study using the same DREADDs approach found the opposite result [73]. This disparity in findings is possibly a result of the use of different methodological parameters. Thus, the contribution of astrocytes to feeding behaviour remains to be elucidated, along with the role of astrocyte signalling in long-term energy homeostasis. 


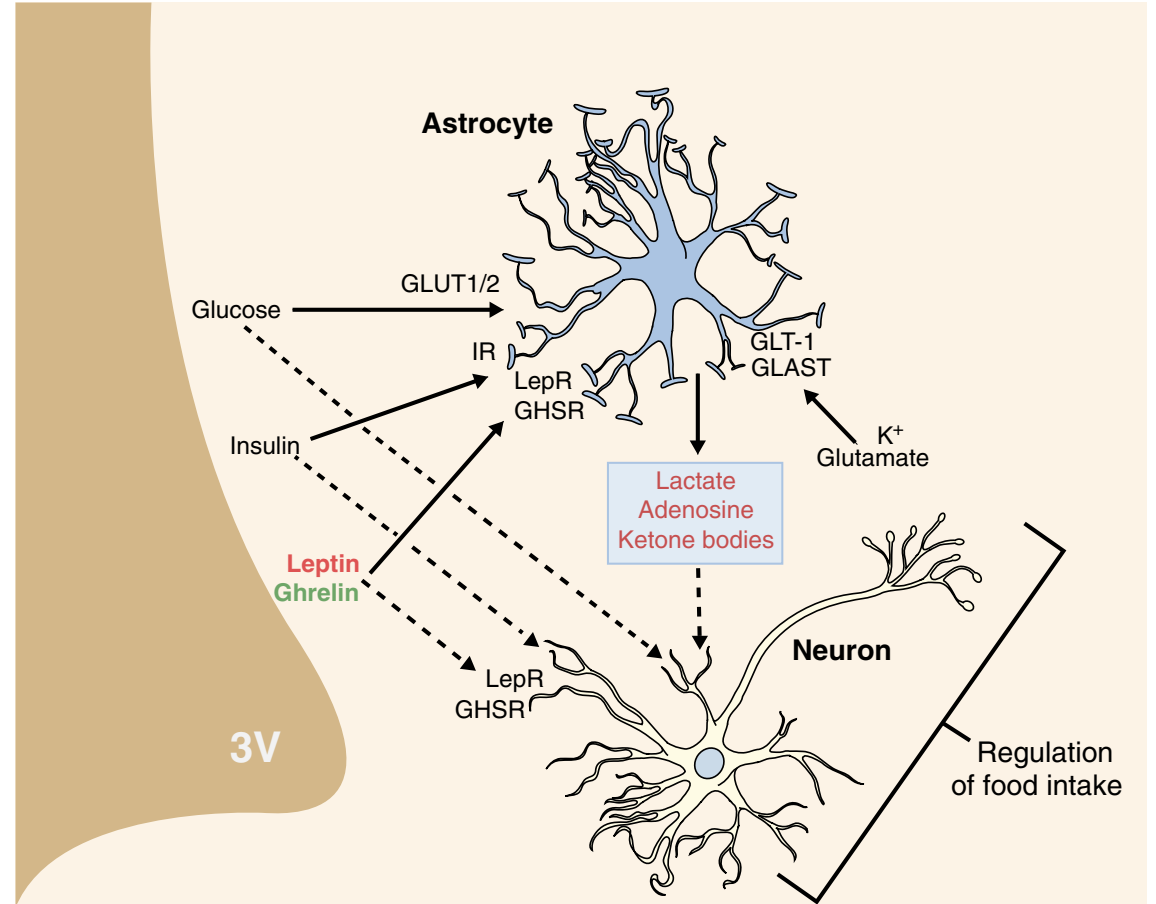

Fig. 2 Astrocytes integrate peripheral nutrient and hormone signals to modulate neuronal activity and function. Astrocytes are critical in maintaining hypothalamic integrity and homeostasis. In addition to regulating neuronal activity through the provision of metabolic substrates and neurotransmitter reuptake, these cells participate in the acute regulation of food intake by peripheral energy cues, such as leptin and ghrelin (green text, increase; red text, decrease). They do this via cognate receptors on their cell surface, including the leptin receptor (LepR) and the growth hormone secretagogue receptor (GHSR) (solid arrow, action on astrocytes; dashed arrow, action on neurons). Astrocytes also contribute to glucose homeostasis regulation through coordination of CNS metabolism. Under HFD feeding or obese conditions, astrocytes become reactive and may contribute to hypothalamic dysfunction and hyperphagia (not shown). $3 \mathrm{~V}$, third ventricle; GLAST, glutamate aspartate transporter; GLT-1, glutamate transporter 1; IR, insulin receptor

inhibition of astrocyte inflammatory signalling has only minimal effects on DIO, paradoxically potentiating the early hyperphagic response to HFD [75]. These data are consistent with the possibility that astrocytic inflammation initially serves as an adaptive response to HFD feeding, limiting neuronal stress and restraining energy intake. With sustained HFD exposure, however, the prolonged gliosis (both microglial and astrocytic) likely promotes neuronal dysfunction and DIO, consistent with its role in a variety of CNS diseases [53].

\section{Tanycytes as nutritional conduits and neuron progenitors}

Tanycytes: barrier and conduit cells of the CNS Tanycytes are specialised ependymal cells found in the lateral walls and floor of the third ventricle. They can be classified into alpha and beta subtypes, each with regional and potentially functional differences [29, 83]. These elongated glial cells simultaneously serve as barrier and conduit cells, with one side of the cell being in contact with the cerebral spinal fluid (CSF) and peripheral circulation through fenestrated capillaries, and the 
other side being in contact with the hypothalamic parenchyma (Fig. 3a). Thus, tanycytes are ideally positioned to sense and transport nutrients and hormones into the brain, and influence the activity of hypothalamic neurons that regulate energy homeostasis.

Tanycytes and metabolic regulation Tanycytes are thought to participate in CNS glucoregulation, partly because of their ability to import glucose across the third ventricle and BBB via GLUT1 transporters on their end-feet (Fig. 3a) [84]. Further, tanycytes may act as glucose sensors via lowaffinity GLUT2 receptors and glucokinase [85, 86], relaying signals to critical glucose-responsive neurons in the hypothalamic arcuate (ARC) and the ventromedial nucleus (VMN) through lactate release [87]. Interestingly, tanycytes also participate in the metabolic adaptation to fasting, allowing increased access of circulating nutrients and hormones into the hypothalamic parenchyma to regulate refeeding hyperphagia
(Fig. 3a) [88]. This gatekeeping function has been recently demonstrated to regulate the uptake of leptin into the hypothalamus through the expression of the leptin receptor on tanycytes of the median eminence (Fig. 3a) [89]. Similarly, it is proposed that tanycytes may regulate transport of other metabolic hormones, though further studies are needed to confirm this.

Tanycyte neurogenesis and obesity In addition to these gatekeeping functions, alpha and beta tanycytes are also considered a key source of stem cells in the adult hypothalamus (Fig. 3a) [90, 91]. Interestingly, beta-2 tanycyte neurogenesis in the median eminence is increased with HFD exposure [90]. Moreover, selective inhibition of this process using targeted radiation blunted weight gain in HFD-fed mice, implicating a role for tanycyte proliferation in obesity [90]. However, in light of the evidence from mice and human-based studies, that hypothalamic
Fig. 3 Tanycytes and NG2 glia contribute to the regulation of energy metabolism. Both tanycytes and polydendrocytes provide BBB stability at the median eminence, are local sources of neural progenitors and regulate leptin signalling. In addition to these common properties, the two cell types have several unique functions. (a)

Tanycytes directly regulate access of circulating hormones and nutrients into the brain parenchyma. (b) NG2 glia are important precursors of oligodendrocytes and provide trophic support to protect and maintain the processes of leptinresponsive neurons. $3 \mathrm{~V}$, third ventricle; + indicates stimulation; LepR, leptin receptor

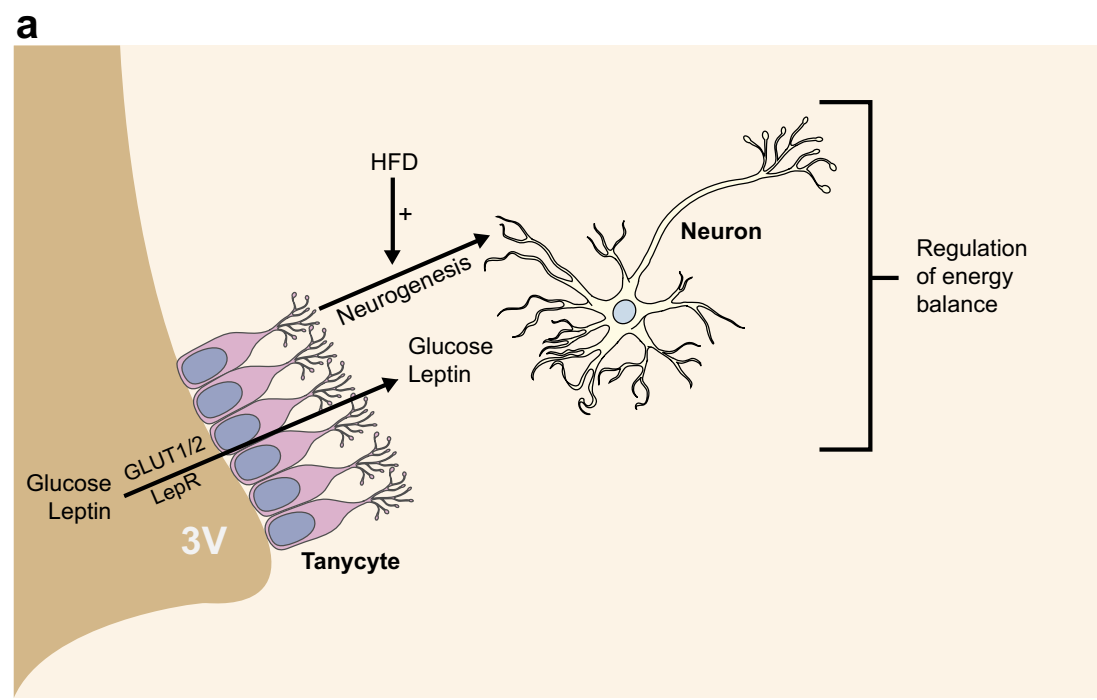

b

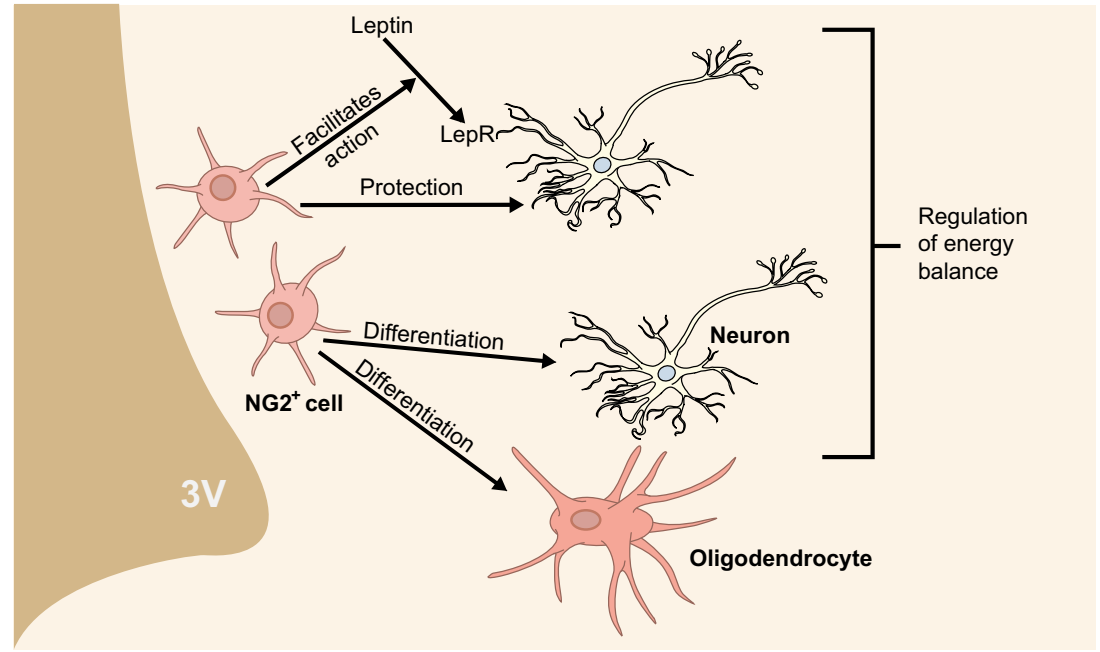


irradiation generally results in hypometabolism and obesity [39, 92], more studies are required to determine whether tanycytes play a causal role in DIO.

\section{NG2 glia and leptin signalling}

NG2 glia: multipotential progenitors of the CNS Polydendrocytes are a recently discovered class of glia that are potentially involved in the hypothalamic control of energy homeostasis. They are characterised by their expression of the nerve/glia antigen 2 (NG2) chondroitin sulphate proteoglycan [93] and are hence also known as NG2 glia (or oligodendrocyte precursor cells [OPC]). These large stellate cells with extensive processes are distributed throughout the CNS. Similar to astrocytes, NG2 glia make contact with neurons and are involved in synaptic transmission, likely via their expression of ion channels and neurotransmitter receptors [94]. NG2 glia are self-renewing and comprise a significant fraction of dividing cells in the adult human brain $[95,96]$. These cells are multipotent progenitors [97], giving rise to new oligodendrocytes (the myelinating glia of the CNS) and neurons [98].

NG2 glia, leptin signalling and obesity While there have been no reports of mature oligodendrocyte contributions to energy homeostasis, a recently published analysis of NG2 glial function in metabolism has revealed an important role in leptin signalling (Fig. 3b) [39]. This study demonstrated that genetic- or irradiation-induced ablation of hypothalamic NG2 glia causes profound obesity in mice, resulting from the degeneration of dendrites from leptin receptor-expressing neurons that extend into the median eminence [39]. Though the mechanisms remain unclear, hypothalamic NG2 glia appear to modulate energy homeostasis both by maintaining the BBB through their proliferative ability, and by preserving neuronal processes that sense leptin and other peripheral energy signals. Furthermore, destruction of NG2 glia may be responsible for hypothalamic dysfunction and weight gain in patients who undergo cancer chemotherapy and cranial irradiation [92]. However, their role in common obesity remains undefined.

\section{Gliosis and obesity in humans}

The impact of obesity on the brain Recent clinical studies have begun to delineate the impact of diet and obesity on the human brain. Broadly, radiologic evidence suggests that obesity is associated with structural alterations to grey and white matter within multiple brain regions that are involved in feeding behaviour and energy homeostasis [99-103]. Moreover, obese patients appear to have reduced integrity of vital brain circuits, correlating with levels of peripheral inflammation and cognitive dysfunction. These findings suggest that an anatomical mechanism may link increased adiposity with CNS impairment [104, 105].

The role of glial cells in human obesity In contrast to their more established role in neurodegenerative disorders, glial cells have not been extensively studied in the pathophysiology of human obesity. Direct comparisons between murine and human glia, particularly microglia and astrocytes, have revealed species-specific differences in morphology and gene expression, but conservation of most biological functions $[106,107]$. These observed differences may have potential significance, as was recently demonstrated by a study involving human astrocyte transplantation into the mouse brain, which resulted in chimeric mice that demonstrated marked improvements in synaptic plasticity and, consequently, superior overall learning capacity [108].

Similar investigations into intra-species differences in the role of glial cells in energy homeostasis remain to be conducted. However, there is indirect evidence supporting glial cell involvement in obesity-associated hypothalamic inflammation in humans; in 2012, we reported a retrospective quantitative analysis of $\mathrm{T} 2$-weighted magnetic resonance images obtained from lean and obese human participants. Remarkably, there was a positive correlation between patient BMI and gliosis within the mediobasal hypothalamus [5]. This radiologic approach paired with immunohistochemical analyses was later applied to mouse models of DIO, demonstrating that the observed changes in T2 signal correlated with histologyconfirmed gliosis and were consistent between rodents and humans [77, 78]. Most recently, a prospective T2-weighted MRI study demonstrated that the degree of hypothalamic gliosis was predictive of BMI and insulin resistance in healthy human participants [79]. Collectively, these findings suggest that hypothalamic gliosis may be functionally linked to obesity in humans and could impact on obesity-related disorders, such as type 2 diabetes and cognitive impairment.

\section{Conclusions}

Despite decades of work on the pathogenesis of obesity, the mechanisms underlying this disease remain elusive. Neurons clearly govern the regulation of energy homeostasis and are obligatory participants in the metabolic changes that result in obesity, but there is new-found interest in the contributions of glia to these processes. Since the initial discovery of HFDinduced hypothalamic inflammation, and its later connection to gliosis, the pace of glial studies has accelerated greatly, revealing glial involvement in many aspects of metabolic 
physiology and pathophysiology. With the new neuroscience tools that have been developed over recent years and adapted for glial research, major advances towards answering the many remaining questions in this research area are imminent. For example, is the gliosis that is associated with HFD feeding strictly pathological or does it represent an adaptive response to environmentally induced neuronal stress? What dietary components drive glial activation during DIO (or are there non-dietary factors involved)? What are the molecular signals produced by glia that alter neuronal activity and the regulation of energy balance? How do tanycytes and NG2 glia contribute to DIO? Is modulation of glial cell function an effective approach for the treatment of obesity? In the coming years, the answers to these and other questions should position glia alongside neurons as equal (rather than silent) partners in energy homeostasis and obesity pathogenesis.

Funding This work was supported by a National Institute of Diabetes and Digestive and Kidney Diseases (NIDDK) postdoctoral fellowship (F32 DK108473) to JDD; a Pilot and Feasibility Award provided by the Nutrition Obesity Research Center (NORC; P30 DK035816) and an American Heart Association (AHA) Scientist Development Grant to MDD; and an American Diabetes Association (ADA) Pathway to Stop Diabetes Grant (1-14-ACE-51) to JPT. In addition, services and support were provided by NORC (DK035816) and the Diabetes Research Center (DK017047) at the University of Washington.

Duality of interest The authors declare that there is no duality of interest associated with this manuscript.

Contribution statement All authors were responsible for drafting the article and revising it critically for important intellectual content. All authors approved the version to be published.

\section{References}

1. Schwartz MW, Woods SC, Porte D Jr, Seeley RJ, Baskin DG (2000) Central nervous system control of food intake. Nature 404:661-671

2. Barsh GS, Farooqi IS, O'Rahilly S (2000) Genetics of bodyweight regulation. Nature 404:644-651

3. Zhang Y, Proenca R, Maffei M, Barone M, Leopold L, Friedman JM (1994) Positional cloning of the mouse obese gene and its human homologue. Nature 372:425-432

4. Burda JE, Sofroniew MV (2014) Reactive gliosis and the multicellular response to CNS damage and disease. Neuron 81:229248

5. Thaler JP, Yi CX, Schur EA et al (2012) Obesity is associated with hypothalamic injury in rodents and humans. J Clin Invest 122: 153-162

6. Valdearcos M, Robblee MM, Benjamin DI, Nomura DK, Xu AW, Koliwad SK (2014) Microglia dictate the impact of saturated fat consumption on hypothalamic inflammation and neuronal function. Cell Rep 9:2124-2138
7. Valdearcos M, Xu AW, Koliwad SK (2015) Hypothalamic inflammation in the control of metabolic function. Annu Rev Physiol 77: $131-160$

8. Thaler JP, Guyenet SJ, Dorfman MD, Wisse BE, Schwartz MW (2013) Hypothalamic inflammation: marker or mechanism of obesity pathogenesis? Diabetes 62:2629-2634

9. Morton GJ, Cummings DE, Baskin DG, Barsh GS, Schwartz MW (2006) Central nervous system control of food intake and body weight. Nature 443:289-295

10. De Souza CT, Araujo EP, Bordin S et al (2005) Consumption of a fat-rich diet activates a proinflammatory response and induces insulin resistance in the hypothalamus. Endocrinology 146: 4192-4199

11. Hotamisligil GS (2006) Inflammation and metabolic disorders. Nature 444:860-867

12. Shoelson SE, Lee J, Goldfine AB (2006) Inflammation and insulin resistance. J Clin Invest 116:1793-1801

13. Schenk S, Saberi M, Olefsky JM (2008) Insulin sensitivity: modulation by nutrients and inflammation. J Clin Invest 118:2992 3002

14. Li JM, Ge CX, Xu MX et al (2015) Betaine recovers hypothalamic neural injury by inhibiting astrogliosis and inflammation in fructose-fed rats. Mol Nutr Food Res 59:189-202

15. Grayson BE, Levasseur PR, Williams SM, Smith MS, Marks DL, Grove KL (2010) Changes in melanocortin expression and inflammatory pathways in fetal offspring of nonhuman primates fed a high-fat diet. Endocrinology 151:1622-1632

16. Posey KA, Clegg DJ, Printz RL et al (2009) Hypothalamic proinflammatory lipid accumulation, inflammation, and insulin resistance in rats fed a high-fat diet. Am J Physiol Endocrinol Metab 296:E1003-E1012

17. Milanski M, Degasperi G, Coope A et al (2009) Saturated fatty acids produce an inflammatory response predominantly through the activation of TLR4 signaling in hypothalamus: implications for the pathogenesis of obesity. J Neurosci 29:359-370

18. Morselli E, Fuente-Martin E, Finan B et al (2014) Hypothalamic PGC-1alpha protects against high-fat diet exposure by regulating ERalpha. Cell Rep 9:633-645

19. Zhang X, Zhang G, Zhang H, Karin M, Bai H, Cai D (2008) Hypothalamic IKKbeta/NF-kappaB and ER stress link overnutrition to energy imbalance and obesity. Cell 135:61-73

20. Munzberg H, Flier JS, Bjorbaek C (2004) Region-specific leptin resistance within the hypothalamus of diet-induced obese mice. Endocrinology 145:4880-4889

21. Kleinridders A, Schenten D, Konner AC et al (2009) MyD88 signaling in the CNS is required for development of fatty acidinduced leptin resistance and diet-induced obesity. Cell Metab 10: 249-259

22. Hong J, Stubbins RE, Smith RR, Harvey AE, Nunez NP (2009) Differential susceptibility to obesity between male, female and ovariectomized female mice. Nutr J 8:11

23. Gao Y, Ottaway N, Schriever SC et al (2014) Hormones and diet, but not body weight, control hypothalamic microglial activity. Glia 62:17-25

24. Garcia-Caceres C, Fuente-Martin E, Diaz F et al (2014) The opposing effects of ghrelin on hypothalamic and systemic inflammatory processes are modulated by its acylation status and food intake in male rats. Endocrinology 155:2868-2880

25. Wang X, Ge A, Cheng M et al (2012) Increased hypothalamic inflammation associated with the susceptibility to obesity in rats exposed to high-fat diet. Exp Diabetes Res 2012:847246

26. Barres BA (2008) The mystery and magic of glia: a perspective on their roles in health and disease. Neuron 60:430-440

27. Pfrieger FW, Slezak M (2012) Genetic approaches to study glial cells in the rodent brain. Glia 60:681-701 
28. Wieghofer P, Prinz M (2016) Genetic manipulation of microglia during brain development and disease. Biochim Biophys Acta 1862:299-309

29. Goodman T, Hajihosseini MK (2015) Hypothalamic tanycytesmasters and servants of metabolic, neuroendocrine, and neurogenic functions. Front Neurosci 9:387

30. Fenno L, Yizhar O, Deisseroth K (2011) The development and application of optogenetics. Annu Rev Neurosci 34:389-412

31. Sternson SM, Roth BL (2014) Chemogenetic tools to interrogate brain functions. Annu Rev Neurosci 37:387-407

32. Yang L, Qi Y, Yang Y (2015) Astrocytes control food intake by inhibiting AGRP neuron activity via adenosine A1 receptors. Cell Rep 11:798-807

33. Ransohoff RM, Cardona AE (2010) The myeloid cells of the central nervous system parenchyma. Nature 468:253-262

34. Ginhoux F, Greter M, Leboeuf M et al (2010) Fate mapping analysis reveals that adult microglia derive from primitive macrophages. Science 330:841-845

35. Ajami B, Bennett JL, Krieger C, McNagny KM, Rossi FM (2011) Infiltrating monocytes trigger EAE progression, but do not contribute to the resident microglia pool. Nat Neurosci 14:1142-1149

36. Hickman SE, Kingery ND, Ohsumi TK et al (2013) The microglial sensome revealed by direct RNA sequencing. Nat Neurosci 16:1896-1905

37. Glass CK, Saijo K, Winner B, Marchetto MC, Gage FH (2010) Mechanisms underlying inflammation in neurodegeneration. Cell 140:918-934

38. Baufeld C, Osterloh A, Prokop S, Miller KR, Heppner FL (2016) High-fat diet-induced brain region-specific phenotypic spectrum of CNS resident microglia. Acta Neuropathol 132:361-375

39. Djogo T, Robins SC, Schneider S et al (2016) Adult NG2-Glia are required for median eminence-mediated leptin sensing and body weight control. Cell Metab 23:797-810

40. Reis WL, Yi CX, Gao Y, Tschop MH, Stern JE (2015) Brain innate immunity regulates hypothalamic arcuate neuronal activity and feeding behavior. Endocrinology 156:1303-1315

41. Sheridan GK, Murphy KJ (2013) Neuron-glia crosstalk in health and disease: fractalkine and CX3CR1 take centre stage. Open Biol 3:130181

42. Wolf Y, Yona S, Kim KW, Jung S (2013) Microglia, seen from the CX3CR1 angle. Front Cell Neurosci 7:26

43. Cardona AE, Pioro EP, Sasse ME et al (2006) Control of microglial neurotoxicity by the fractalkine receptor. Nat Neurosci 9:917-924

44. Morris DL, Oatmen KE, Wang T, DelProposto JL, Lumeng CN (2012) CX3CR1 deficiency does not influence trafficking of adipose tissue macrophages in mice with diet-induced obesity. Obesity 20:1189-1199

45. Polyak A, Ferenczi S, Denes A et al (2014) The fractalkine/ Cx3CR1 system is implicated in the development of metabolic visceral adipose tissue inflammation in obesity. Brain Behav Immun 38:25-35

46. Morari J, Anhe GF, Nascimento LF et al (2014) Fractalkine (CX3CL1) is involved in the early activation of hypothalamic inflammation in experimental obesity. Diabetes 63:3770-3784

47. Shah R, O'Neill SM, Hinkle C et al (2015) Metabolic effects of CX3CR1 deficiency in diet-induced obese mice. PLoS One 10: e0138317

48. Lee YS, Morinaga H, Kim JJ et al (2013) The fractalkine/ CX3CR1 system regulates beta cell function and insulin secretion. Cell 153:413-425

49. Imai T, Hieshima K, Haskell $\mathrm{C}$ et al (1997) Identification and molecular characterization of fractalkine receptor CX3CR1, which mediates both leukocyte migration and adhesion. Cell 91: 521-530
50. Jung S, Aliberti J, Graemmel P et al (2000) Analysis of fractalkine receptor CX(3)CR1 function by targeted deletion and green fluorescent protein reporter gene insertion. Mol Cell Biol 20:41064114

51. Butovsky O, Jedrychowski MP, Moore CS et al (2014) Identification of a unique TGF-beta-dependent molecular and functional signature in microglia. Nat Neurosci 17:131-143

52. Zhang Y, Chen K, Sloan SA et al (2014) An RNA-sequencing transcriptome and splicing database of glia, neurons, and vascular cells of the cerebral cortex. J Neurosci 34:11929-11947

53. Sofroniew MV, Vinters HV (2010) Astrocytes: biology and pathology. Acta Neuropathol 119:7-35

54. Garcia-Caceres C, Quarta C, Varela L et al (2016) Astrocytic insulin signaling couples brain glucose uptake with nutrient availability. Cell 166:867-880

55. Rothstein JD, Dykes-Hoberg M, Pardo CA et al (1996) Knockout of glutamate transporters reveals a major role for astroglial transport in excitotoxicity and clearance of glutamate. Neuron 16:675686

56. Cataldo AM, Broadwell RD (1986) Cytochemical identification of cerebral glycogen and glucose-6-phosphatase activity under normal and experimental conditions. II. Choroid plexus and ependymal epithelia, endothelia and pericytes. J Neurocytol 15: 511-524

57. Leloup C, Arluison M, Lepetit N et al (1994) Glucose transporter 2 (GLUT 2): expression in specific brain nuclei. Brain Res 638: 221-226

58. Suh SW, Bergher JP, Anderson CM, Treadway JL, Fosgerau K, Swanson RA (2007) Astrocyte glycogen sustains neuronal activity during hypoglycemia: studies with the glycogen phosphorylase inhibitor CP-316,819 ([R-R*, S*]-5-chloro-N-[2-hydroxy-3(methoxymethylamino)-3-oxo-1-(phenylmethyl)pro pyl]-1H-indole-2-carboxamide). J Pharmacol Exp Ther 321:45-50

59. Brown AM, Sickmann HM, Fosgerau K et al (2005) Astrocyte glycogen metabolism is required for neural activity during aglycemia or intense stimulation in mouse white matter. J Neurosci Res 79:74-80

60. Brown AM, Tekkok SB, Ransom BR (2003) Glycogen regulation and functional role in mouse white matter. J Physiol 549:501-512

61. Newman LA, Korol DL, Gold PE (2011) Lactate produced by glycogenolysis in astrocytes regulates memory processing. PLoS One 6:e28427

62. Suzuki A, Stern SA, Bozdagi O et al (2011) Astrocyte-neuron lactate transport is required for long-term memory formation. Cell 144:810-823

63. Le Foll C, Dunn-Meynell AA, Miziorko HM, Levin BE (2014) Regulation of hypothalamic neuronal sensing and food intake by ketone bodies and fatty acids. Diabetes 63:1259-1269

64. Le Foll C, Levin BE (2016) Fatty acid-induced astrocyte ketone production and the control of food intake. Am J Physiol Regul Integr Comp Physiol 310:R1186-R1192

65. Cheunsuang O, Morris R (2005) Astrocytes in the arcuate nucleus and median eminence that take up a fluorescent dye from the circulation express leptin receptors and neuropeptide Y Y1 receptors. Glia 52:228-233

66. Fuente-Martin E, Garcia-Caceres C, Granado M et al (2012) Leptin regulates glutamate and glucose transporters in hypothalamic astrocytes. J Clin Invest 122:3900-3913

67. Kim JG, Suyama S, Koch M et al (2014) Leptin signaling in astrocytes regulates hypothalamic neuronal circuits and feeding. Nat Neurosci 17:908-910

68. Rottkamp DM, Rudenko IA, Maier MT et al (2015) Leptin potentiates astrogenesis in the developing hypothalamus. Mol Metab 4: 881-889 
69. Hsuchou H, He Y, Kastin AJ et al (2009) Obesity induces functional astrocytic leptin receptors in hypothalamus. Brain: J Neurol 132:889-902

70. Jayaram B, Pan W, Wang Y et al (1985) (2013) Astrocytic leptinreceptor knockout mice show partial rescue of leptin resistance in diet-induced obesity. J Appl Physiol 114:734-741

71. Fuente-Martin E, Garcia-Caceres C, Argente-Arizon P et al (2016) Ghrelin regulates glucose and glutamate transporters in hypothalamic astrocytes. Sci Rep 6:23673

72. Reiner DJ, Mietlicki-Baase EG, McGrath LE et al (2016) Astrocytes regulate GLP-1 receptor-mediated effects on energy balance. J Neurosci 36:3531-3540

73. Chen N, Sugihara H, Kim J et al (2016) Direct modulation of GFAP-expressing glia in the arcuate nucleus bi-directionally regulates feeding. Elife 5, e18716

74. Guyenet SJ, Nguyen HT, Hwang BH, Schwartz MW, Baskin DG, Thaler JP (2013) High-fat diet feeding causes rapid, non-apoptotic cleavage of caspase-3 in astrocytes. Brain Res 1512:97-105

75. Buckman LB, Thompson MM, Lippert RN, Blackwell TS, Yull FE, Ellacott KL (2015) Evidence for a novel functional role of astrocytes in the acute homeostatic response to high-fat diet intake in mice. Mol Metab 4:58-63

76. Buckman LB, Thompson MM, Moreno HN, Ellacott KL (2013) Regional astrogliosis in the mouse hypothalamus in response to obesity. J Comp Neurol 521:1322-1333

77. Berkseth KE, Guyenet SJ, Melhorn SJ et al (2014) Hypothalamic gliosis associated with high-fat diet feeding is reversible in mice: a combined immunohistochemical and magnetic resonance imaging study. Endocrinology 155:2858-2867

78. Lee D, Thaler JP, Berkseth KE, Melhorn SJ, Schwartz MW, Schur EA (2013) Longer T(2) relaxation time is a marker of hypothalamic gliosis in mice with diet-induced obesity. Am J Physiol Endocrinol Metab 304:E1245-E1250

79. Schur EA, Melhorn SJ, Oh SK et al (2015) Radiologic evidence that hypothalamic gliosis is associated with obesity and insulin resistance in humans. Obesity 23:2142-2148

80. Yi CX, Gericke M, Kruger M et al (2012) High calorie diet triggers hypothalamic angiopathy. Mol Metab 1:95-100

81. Bull C, Freitas KC, Zou S et al (2014) Rat nucleus accumbens core astrocytes modulate reward and the motivation to self-administer ethanol after abstinence. Neuropsychopharmacol 39:2835-2845

82. Horvath TL, Sarman B, Garcia-Caceres C et al (2010) Synaptic input organization of the melanocortin system predicts dietinduced hypothalamic reactive gliosis and obesity. Proc Natl Acad Sci U S A 107:14875-14880

83. Elizondo-Vega R, Cortes-Campos C, Barahona MJ, Oyarce KA, Carril CA, Garcia-Robles MA (2015) The role of tanycytes in hypothalamic glucosensing. J Cell Mol Med 19:1471-1482

84. Morgello S, Uson RR, Schwartz EJ, Haber RS (1995) The human blood-brain barrier glucose transporter (GLUT1) is a glucose transporter of gray matter astrocytes. Glia 14:43-54

85. Garcia M, Millan C, Balmaceda-Aguilera C et al (2003) Hypothalamic ependymal-glial cells express the glucose transporter GLUT2, a protein involved in glucose sensing. J Neurochem 86:709-724

86. Millan C, Martinez F, Cortes-Campos C et al (2010) Glial glucokinase expression in adult and post-natal development of the hypothalamic region. ASN Neuro 2:e00035

87. Cortes-Campos C, Elizondo R, Llanos P, Uranga RM, Nualart F, Garcia MA (2011) MCT expression and lactate influx/efflux in tanycytes involved in glia-neuron metabolic interaction. PLoS One 6:e16411

88. Langlet F, Levin BE, Luquet S et al (2013) Tanycytic VEGF-A boosts blood-hypothalamus barrier plasticity and access of metabolic signals to the arcuate nucleus in response to fasting. Cell Metab 17:607-617

89. Balland E, Dam J, Langlet F et al (2014) Hypothalamic tanycytes are an ERK-gated conduit for leptin into the brain. Cell Metab 19: 293-301

90. Lee DA, Bedont JL, Pak T et al (2012) Tanycytes of the hypothalamic median eminence form a diet-responsive neurogenic niche. Nat Neurosci 15:700-702

91. Robins SC, Stewart I, McNay DE et al (2013) Alpha-Tanycytes of the adult hypothalamic third ventricle include distinct populations of FGF-responsive neural progenitors. Nat Commun 4:2049

92. Iughetti L, Bruzzi P, Predieri B, Paolucci P (2012) Obesity in patients with acute lymphoblastic leukemia in childhood. Ital J Pediatr 38:4

93. Levine JM, Stallcup WB (1987) Plasticity of developing cerebellar cells in vitro studied with antibodies against the NG2 antigen. J Neurosci 7:2721-2731

94. Dimou L, Gallo V (2015) NG2-glia and their functions in the central nervous system. Glia 63:1429-1451

95. Rhee W, Ray S, Yokoo H et al (2009) Quantitative analysis of mitotic Olig2 cells in adult human brain and gliomas: implications for glioma histogenesis and biology. Glia 57:510-523

96. Geha S, Pallud J, Junier MP et al (2010) NG2+/Olig2+ cells are the major cycle-related cell population of the adult human normal brain. Brain Pathol 20:399-411

97. Nishiyama A, Komitova M, Suzuki R, Zhu X (2009) Polydendrocytes (NG2 cells): multifunctional cells with lineage plasticity. Nat Rev Neurosci 10:9-22

98. Robins SC, Trudel E, Rotondi O et al (2013) Evidence for NG2glia derived, adult-born functional neurons in the hypothalamus. PLoS One 8:e78236

99. Mueller K, Anwander A, Moller HE et al (2011) Sex-dependent influences of obesity on cerebral white matter investigated by diffusion-tensor imaging. PLoS One 6:e18544

100. Stanek KM, Grieve SM, Brickman AM et al (2011) Obesity is associated with reduced white matter integrity in otherwise healthy adults. Obesity 19:500-504

101. Karlsson HK, Tuulari JJ, Hirvonen J et al (2013) Obesity is associated with white matter atrophy: a combined diffusion tensor imaging and voxel-based morphometric study. Obesity 21:2530 2537

102. Yokum S, Ng J, Stice E (2012) Relation of regional gray and white matter volumes to current BMI and future increases in BMI: a prospective MRI study. Int J Obes 36:656-664

103. Shott ME, Cornier MA, Mittal VA et al (2015) Orbitofrontal cortex volume and brain reward response in obesity. Int J Obes 39: 214-221

104. Puig J, Blasco G, Daunis IEJ et al (2015) Hypothalamic damage is associated with inflammatory markers and worse cognitive performance in obese subjects. J Clin Endocrinol Metab 100:E276E281

105. Cazettes F, Cohen JI, Yau PL, Talbot H, Convit A (2011) Obesitymediated inflammation may damage the brain circuit that regulates food intake. Brain Res 1373:101-109

106. Smith AM, Dragunow M (2014) The human side of microglia. Trends Neurosci 37:125-135

107. Zhang Y, Sloan SA, Clarke LE et al (2016) Purification and characterization of progenitor and mature human astrocytes reveals transcriptional and functional differences with mouse. Neuron 89:37-53

108. Han X, Chen M, Wang F et al (2013) Forebrain engraftment by human glial progenitor cells enhances synaptic plasticity and learning in adult mice. Cell Stem Cell 12:342-353 